\title{
Association between Influenza-like IIInesses and Social Determinants of Health by Census Tract in Houston/ Harris County
}

\author{
Biru Yang*, Nathan Wang, Wesley McNeely, Salma Khuwaja and Raouf Arafat \\ Houston Department of Health and Human Services, Houston, TX, USA
}

\section{Objective}

To investigate the association between social determinants of health and influenza-like illnesses in Houston/Harris County and to identify neighborhoods for targeted surveillance or interventions.

\section{Introduction}

The Houston Department of Health Department of Health and Human Services (HDHHS) monitors emergency departments (ED) chief complaints across the Houston metropolitan area, Harris County, and the surrounding jurisdictions by Real-time Outbreak Disease Surveillance (RODS). The influenza-like illnesses (ILI) data is collected by sentinel surveillance provider network of 12 physicians and RODS, an electronic syndromic surveillance database consisting of about 30 EDs in metropolitan Houston. Previous research indicates that there is a relationship between new HIV diagnoses and neighborhood poverty. However, there is limited research on health disparity to investigate the association between influenza-like illnesses (ILI) and social determinants of health (SDH), such as poverty.

\section{Methods}

This is a cross-sectional study which investigates the relationship between ILI and SDH. ILI cases will be linked to Census variables using residential address at influenza diagnosis. Sex, age, and race/ ethnicity are considered in the analysis. RODS information are used to identify areas where more public health interventions are needed. A case of ILI is defined as having at least one respiratory illness symptom (cough, sore throat, or chills) and fever. Influenza laboratory data is provided by HDHHS laboratory. RODS v.4.2 is used as the syndromic surveillance system. Oracle SQL Developer v.3.2 is used to extract data from the RODS system. Cases are geocoded and analyzed at the census tract level and published only in an aggregate form. Poverty is defined as percent below poverty which was categorized into four groups (below 5\%, 5-10\%, 10-20\%, and more than $20 \%$ below the poverty line).

\section{Results}

Analyses are being conducted to ascertain the associations between ILI and SDH. RODS data corresponded with lab data during the previous 4 influenza seasons tracked healthcare seeking behaviors related to the $2009 \mathrm{H} 1 \mathrm{~N} 1$ strain, the reduced severity of the 2010-2011 strains, and the increase in healthcare seeking behavior before flu in 2011-2012. For the 2011-2012 influenza season, RODS identified that while the majority of ILI symptoms came from patients living in the area west of Houston, the majority ( 8 of 12) of sentinel providers was located in areas 0-2 standard deviations below the norm.

\section{Conclusions}

Syndromic surveillance can help determine the severity of influenza during a season, and indicate the presence of other respiratory-related diseases. It identifies future areas to consider when enrolling new influenza sentinel surveillance providers: SE Houston, NE Houston, and North Houston. Coupled with census data, public health departments can allocate resources to areas that are most impacted by SDH variables such as poverty. Additional conclusions are pending the completion of the analysis of SDH and ILI surveillance data.

\section{Keywords}

Health Disparity; Syndromic Surveillance; Influenza-Like illness; Geographic Analysis

\section{Acknowledgments}

This work was supported by the Houston Department of Health and Human Services Bureau of Epidemiology through a grant from the Department of Homeland Security Urban Area Security Initiative.

\section{References}

1. Harvard Schoolof Public Health (2004) Public Health Disparities Geocoding Project. Available at http://www.hsph.harvard.edu/thegeocodingproject/

2. Yousey-Hindes, K. T. (April, 2012). Guidance for Calculating Incidence by Census Tract Poverty Level Using 2010 Census and 20062010 ACS. Retrieved October 31, 2012, from http://www.cste.org: http://www.cste.org/webpdfs/AreabasedPovertyCensus2010Demov3. pdf

\footnotetext{
*Biru Yang

E-mail: biru.yang@houstontx.gov
} 